International Journal of Pure and Applied Mathematics

Volume 94 No. $4 \quad 2014,477-487$

ISSN: 1311-8080 (printed version); ISSN: 1314-3395 (on-line version)

url: http://www.ijpam.eu

doi: http://dx.doi.org/10.12732/ijpam.v94i4.3

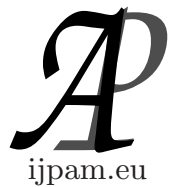

\title{
DELAY-DEPENDENT OUTPUT FEEDBACK GUARANTEED COST CONTROL FOR HOPFIELD NEURAL NETWORKS WITH INTERVAL TIME-VARYING DELAY
}

\author{
Manlika Rajchakit ${ }^{1}$, Grienggrai Rajchakit ${ }^{2} \S$ \\ Department of Mathematics and Statistics \\ Maejo University \\ Chiangmai, 50290, THAILAND
}

\begin{abstract}
This paper studies the problem of guaranteed cost control for a class of Hopfield delayed neural networks. The time delay is a continuous function belonging to a given interval, but not necessary to be differentiable. A cost function is considered as a nonlinear performance measure for the closed-loop system. The stabilizing controllers to be designed must satisfy some exponential stability constraints on the closed-loop poles. By constructing a set of augmented Lyapunov-Krasovskii functional combined with Newton-Leibniz formula, a guaranteed cost controller is designed via memoryless state feedback control and new sufficient conditions for the existence of the guaranteed cost state-feedback for the system are given in terms of linear matrix inequalities (LMIs).
\end{abstract}

AMS Subject Classification: 92B05, 93D20, 37C75

Key Words: Hopfield neural networks, guaranteed cost control, stabilization, interval time-varying delays, Lyapunov function, linear matrix inequalities

\section{Introduction}

Recently, dynamical recurrent neural networks have attracted considerable at-

Received: January 16, 2014

(C) 2014 Academic Publications, Ltd.

$\S$ Correspondence author url: www.acadpubl.eu 
tentions as they are proved to be essential in applications such as classification of patterns, associative memories, and optimization. In particular, the recurrent neural network model introduced by Hopfield has been studied extensively and has been successfully applied to optimization problems. Moreover, time delay is commonly encountered in the implementation of neural networks due to the finite speed of information processing, and it is frequently a source of oscillation and instability in neural networks. Therefore, the stability of delayed neural networks has become a topic of great theoretical and practical importance. In many practical systems, it is desirable to design neural networks which are not only asymptotically or exponentially stable but can also guarantee an adequate level of system performance. In the area of control, signal processing, pattern recognition and image processing, delayed neural networks have many useful applications. Some of these applications require that the equilibrium points of the designed network be stable. In both biological and artificial neural systems, time delays due to integration and communication are ubiquitous and often become a source of instability. The time delays in electronic neural networks are usually time-varying, and sometimes vary violently with respect to time due to the finite switching speed of amplifiers and faults in the electrical circuitry. Guaranteed cost control problem [1-9] has the advantage of providing an upper bound on a given system performance index and thus the system performance degradation incurred by the uncertainties or time delays is guaranteed to be less than this bound. The Lyapunov-Krasovskii functional technique has been among the popular and effective tool in the design of guaranteed cost controls for neural networks with time delay. Nevertheless, despite such diversity of results available, most existing work either assumed that the time delays are constant or differentiable [4-7]. Although, in some cases, delay-dependent guaranteed cost control for systems with time-varying delays were considered in $[2$, 4, 7-9], the approach used there can not be applied to systems with interval, non-differentiable time-varying delays. To the best of our knowledge, the guaranteed cost control and state feedback stabilization for Hopfield neural networks with interval, non-differentiable time-varying delays have not been fully studied yet, which are important in both theories and applications. This motivates our research.

In this paper, we investigate the guaranteed cost control for Hopfield delayed neural networks problem. The novel features here are that the delayed neural network under consideration is with various globally Lipschitz continuous activation functions, and the time-varying delay function is interval, nondifferentiable. A nonlinear cost function is considered as a performance measure for the closed-loop system. The stabilizing controllers to be designed must sat- 
isfy some exponential stability constraints on the closed-loop poles. Based on constructing a set of augmented Lyapunov-Krasovskii functional combined with Newton-Leibniz formula, new delay-dependent criteria for guaranteed cost control via memoryless feedback control is established in terms of LMIs, which allow simultaneous computation of two bounds that characterize the exponential stability rate of the solution.

The outline of the paper is as follows. Section 2 presents definitions and some well-known technical propositions needed for the proof of the main result. LMI delay-dependent criteria for guaranteed cost control is presented in Section 3. The paper ends with conclusions and cited references.

\section{Preliminaries}

The following notation will be used in this paper. $\mathbb{R}^{+}$denotes the set of all real non-negative numbers; $\mathbb{R}^{n}$ denotes the $n$-dimensional space with the scalar product $\langle x, y\rangle$ or $x^{T} y$ of two vectors $x, y$, and the vector norm $\|$. $\|$; $M^{n \times r}$ denotes the space of all matrices of $(n \times r)$-dimensions. $A^{T}$ denotes the transpose of matrix $A ; A$ is symmetric if $A=A^{T}$; $I$ denotes the identity matrix; $\lambda(A)$ denotes the set of all eigenvalues of $A ; \lambda_{\max }(A)=\max \{\operatorname{Re} \lambda ; \lambda \in \lambda(A)\}$. $x_{t}:=\{x(t+s): s \in[-h, 0]\},\left\|x_{t}\right\|=\sup _{s \in[-h, 0]}\|x(t+s)\| ; C^{1}\left([0, t], \mathbb{R}^{n}\right)$ denotes the set of all $\mathbb{R}^{n}$-valued continuously differentiable functions on $[0, t]$; $L_{2}\left([0, t], \mathbb{R}^{m}\right)$ denotes the set of all the $\mathbb{R}^{m}$-valued square integrable functions on $[0, t]$;

Matrix $A$ is called semi-positive definite $(A \geq 0)$ if $\langle A x, x\rangle \geq 0$, for all $x \in \mathbb{R}^{n} ; A$ is positive definite $(A>0)$ if $\langle A x, x\rangle>0$ for all $x \neq 0 ; A>B$ means $A-B>0$. The notation $\operatorname{diag}\{\ldots\}$ stands for a block-diagonal matrix. The symmetric term in a matrix is denoted by $*$.

Consider the following Hopfield neural networks with interval time-varying delay:

$$
\begin{aligned}
& \dot{x}(t)=-A x(t)+W_{0} f(x(t))+W_{1} g(x(t-h(t)))+B u(t), \quad t \geq 0, \\
& x(t)=\phi(t), t \in\left[-h_{1}, 0\right],
\end{aligned}
$$

where $x(t)=\left[x_{1}(t), x_{2}(t), \ldots, x_{n}(t)\right]^{T} \in \mathbb{R}^{n}$ is the state of the neural, $u(.) \in$ $L_{2}\left([0, t], \mathbb{R}^{m}\right)$ is the control; $n$ is the number of neurals, and

$$
\begin{aligned}
& f(x(t))=\left[f_{1}\left(x_{1}(t)\right), f_{2}\left(x_{2}(t)\right), \ldots, f_{n}\left(x_{n}(t)\right)\right]^{T}, \\
& g(x(t))=\left[g_{1}\left(x_{1}(t)\right), g_{2}\left(x_{2}(t)\right), \ldots, g_{n}\left(x_{n}(t)\right)\right]^{T},
\end{aligned}
$$


are the activation functions; $A=\operatorname{diag}\left(\bar{a}_{1}, \bar{a}_{2}, \ldots, \bar{a}_{n}\right), \bar{a}_{i}>0$ represents the self-feedback term; $B \in R^{n \times m}$ is control input matrix; $W_{0}, W_{1}$ denote the connection weights, the discretely delayed connection weights and the distributively delayed connection weight, respectively; The time-varying delay function $h(t)$ satisfies the condition

$$
0 \leq h_{0} \leq h(t) \leq h_{1},
$$

The initial functions $\phi(t) \in C^{1}\left(\left[-h_{1}, 0\right], R^{n}\right)$, with the norm

$$
\|\phi\|=\sup _{t \in\left[-h_{1}, 0\right]} \sqrt{\|\phi(t)\|^{2}+\|\dot{\phi}(t)\|^{2}} .
$$

In this paper we consider various activation functions and assume that the activation functions $f(),. g($.$) are Lipschitzian with the Lipschitz constants f_{i}, e_{i}>$ 0 :

$$
\begin{aligned}
& \left|f_{i}\left(\xi_{1}\right)-f_{i}\left(\xi_{2}\right)\right| \leq f_{i}\left|\xi_{1}-\xi_{2}\right|, \quad i=1,2, \ldots, n, \forall \xi_{1}, \xi_{2} \in \mathbb{R} \\
& \left|g_{i}\left(\xi_{1}\right)-g_{i}\left(\xi_{2}\right)\right| \leq e_{i}\left|\xi_{1}-\xi_{2}\right|, \quad i=1,2, \ldots, n, \forall \xi_{1}, \xi_{2} \in \mathbb{R}
\end{aligned}
$$

The performance index associate with the system (1) is the following function

$$
J=\int_{0}^{\infty} f^{0}(t, x(t), x(t-h(t)), u(t)) d t,
$$

where $f^{0}(t, x(t), x(t-h(t)), u(t)): R^{+} \times R^{n} \times R^{n} \times R^{m} \rightarrow R^{+}$, is a nonlinear cost function satisfies

$$
\exists Q_{1}, Q_{2}, R: f^{0}(t, x, y, u) \leq\left\langle Q_{1} x, x\right\rangle+\left\langle Q_{2} y, y\right\rangle+\langle R u, u\rangle,
$$

for all $(t, x, u) \in R^{+} \times R^{n} \times R^{m}$ and $Q_{1}, Q_{2} \in R^{n \times n}, R \in R^{m \times m}$, are given symmetric positive definite matrices. The objective of this paper is to design a memoryless state feedback controller $u(t)=K x(t)$ for system (1) and the cost function (3) such that the resulting closed-loop system

$$
\dot{x}(t)=-(A-B K) x(t)+W_{0} f(x(t))+W_{1} g(x(t-h(t))),
$$

is exponentially stable and the closed-loop value of the cost function (3) is minimized.

Definition 1 Given $\alpha>0$. The zero solution of closed-loop system (5) is $\alpha$-exponentially stabilizable if there exist a positive number $N>0$ such that every solution $x(t, \phi)$ satisfies the following condition:

$$
\|x(t, \phi)\| \leq N e^{-\alpha t}\|\phi\|, \quad \forall t \geq 0 .
$$


Definition 2 Consider the control system (1). If there exist a memoryless state feedback control law $u^{*}(t)=K x(t)$ and a positive number $J^{*}$ such that the zero solution of the closed-loop system (5) is exponentially stable and the cost function (3) satisfies $J \leq J^{*}$, then the value $J^{*}$ is a guaranteed costant and $u^{*}(t)$ is a guaranteed cost control law of the system and its corresponding cost function.

We introduce the following technical well-known propositions, which will be used in the proof of our results.

Proposition 1.(Schur complement lemma [10]). Given constant matrices $X, Y, Z$ with appropriate dimensions satisfying $X=X^{T}, Y=Y^{T}>0$. Then $X+Z^{T} Y^{-1} Z<0$ if and only if

$$
\left(\begin{array}{cc}
X & Z^{T} \\
Z & -Y
\end{array}\right)<0
$$

Proposition 2.(Integral matrix inequality [10]). For any symmetric positive definite matrix $M>0$, scalar $\gamma>0$ and vector function $\omega:[0, \gamma] \rightarrow \mathbb{R}^{n}$ such that the integrations concerned are well defined, the following inequality holds

$$
\left(\int_{0}^{\gamma} \omega(s) d s\right)^{T} M\left(\int_{0}^{\gamma} \omega(s) d s\right) \leq \gamma\left(\int_{0}^{\gamma} \omega^{T}(s) M \omega(s) d s\right)
$$

\section{Main Results}

In this section, we give a design of memoryless guaranteed feedback cost control for neural networks (1). Let us set

$$
\begin{aligned}
& W_{11}=-[P+\alpha I] A-A^{T}[P+\alpha I]-2 B B^{T}+0.25 B R B^{T}+\sum_{i=0}^{1} G_{i}, \\
& W_{12}=P+A P+0.5 B B^{T}, W_{13}=e^{-2 \alpha h_{0}} H_{0}+0.5 B B^{T}+A P, \\
& W_{14}=2 e^{-2 \alpha h_{1}} H_{1}+0.5 B B^{T}+A P, W_{15}=P 0.5 B B^{T}+A P, \\
& W_{22}=\sum_{i=0}^{1} W_{i} D_{i} W_{i}^{T}+\sum_{i=0}^{1} h_{i}^{2} H_{i}+\left(h_{1}-h_{0}\right) U-2 P-B B^{T},
\end{aligned}
$$




$$
\begin{aligned}
& W_{23}=P, \quad W_{24}=P, \quad W_{25}=P \\
& W_{33}=-e^{-2 \alpha h_{0}} G_{0}-e^{-2 \alpha h_{0}} H_{0}-e^{-2 \alpha h_{1}} U+\sum_{i=0}^{1} W_{i} D_{i} W_{i}^{T}, \\
& W_{34}=0, \quad W_{35}=-2 \alpha h_{1} U, \\
& W_{44}=\sum_{i=0}^{1} W_{i} D_{i} W_{i}^{T}-e^{-2 \alpha h_{1}} U-e^{-2 \alpha h_{1}} G_{1}-e^{-2 \alpha h_{1}} H_{1}, \quad W_{45}=e^{-2 \alpha h_{1}} U, \\
& W_{55}=-e^{-2 \alpha h_{1}} U+W_{0} D_{0} W_{0}^{T}, E=\operatorname{diag}\left\{e_{i}, i=1, \ldots, n\right\}, \\
& F=\operatorname{diag}\left\{f_{i}, i=1, \ldots, n\right\}, \lambda_{1}=\lambda_{\min }\left(P^{-1}\right), \\
& \lambda_{2}=\lambda_{\max }\left(P^{-1}\right)+h_{0} \lambda_{\max }\left[P^{-1}\left(\sum_{i=0}^{1} G_{i}\right) P^{-1}\right] \\
& \quad+h_{1}^{2} \lambda_{\max }\left[P^{-1}\left(\sum_{i=0}^{1} H_{i}\right) P^{-1}\right]+\left(h_{1}-h_{0}\right) \lambda_{\max }\left(P^{-1} U P^{-1}\right) .
\end{aligned}
$$

Theorem 1. Consider control system (1) and the cost function (3). If there exist symmetric positive definite matrices $P, U, G_{0}, G_{1}, H_{0}, H_{1}$, and diagonal positive definite matrices $D_{i}, i=0,1$ satisfying the following LMIs

$$
\begin{aligned}
& {\left[\begin{array}{ccccl}
W_{11} & W_{12} & W_{13} & W_{14} & W_{15} \\
* & W_{22} & W_{23} & W_{24} & W_{25} \\
* & * & W_{33} & W_{34} & W_{35} \\
* & * & * & W_{44} & W_{45} \\
* & * & * & * & W_{55}
\end{array}\right]<0} \\
& {\left[\begin{array}{ccc}
-P A-A^{T} P-\sum_{i=0}^{1} e^{-2 \alpha h_{i}} H_{i} & 2 P F & P Q_{1} \\
* & -D_{0} & 0 \\
* & * & -Q_{1}^{-1}
\end{array}\right]<0,} \\
& {\left[\begin{array}{ccc}
W_{1} D_{1} W_{1}^{T}-e^{-2 \alpha h_{1}} U & 2 P E & P Q_{2} \\
* & -D_{1} & 0 \\
* & * & -Q_{2}^{-1}
\end{array}\right]<0,}
\end{aligned}
$$

then

$$
u(t)=-\frac{1}{2} B^{T} P^{-1} x(t), \quad t \geq 0 .
$$

is a guaranteed cost control and the guaranteed cost value is given by

$$
J^{*}=\lambda_{2}\|\phi\|^{2}
$$


Moreover, the solution $x(t, \phi)$ of the system satisfies

$$
\|x(t, \phi)\| \leq \sqrt{\frac{\lambda_{1}}{\lambda_{2}}} e^{-\alpha t}\|\phi\|, \quad \forall t \geq 0 .
$$

Proof. Let $Y=P^{-1}, y(t)=Y x(t)$. Using the feddback control (5) we consider the following Lyapunov-Krasovskii functional

$$
\begin{gathered}
V\left(t, x_{t}\right)=\sum_{i=1}^{3} V_{i}\left(t, x_{t}\right), \\
V_{1}=x^{T}(t) Y x(t), \\
V_{2}=\int_{t-h_{0}}^{t} e^{2 \alpha(s-t)} x^{T}(s) Y G_{0} Y x(s) d s, \\
V_{3}=\int_{t-h_{1}}^{t} e^{2 \alpha(s-t)} x^{T}(s) Y G_{1} Y x(s) d s .
\end{gathered}
$$

It easy to check that

$$
\lambda_{1}\|x(t)\|^{2} \leq V\left(t, x_{t}\right) \leq \lambda_{2}\left\|x_{t}\right\|^{2}, \quad \forall t \geq 0
$$

Taking the derivative of $V_{i}, i=1,2,3$ we have

$$
\begin{aligned}
& \dot{V}_{1}=2 x^{T}(t) Y \dot{x}(t) \\
& =y^{T}(t)\left[-P A^{T}-A P\right] y(t)-y^{T}(t) B B^{T} y(t) \\
& +2 y^{T}(t) W_{0} f(.) y(t)+2 y^{T}(t) W_{1} g(.) y(t) \\
& \dot{V}_{2}=y^{T}(t) G_{0} y(t)-e^{-2 \alpha h_{0}} y^{T}\left(t-h_{0}\right) G_{0} y\left(t-h_{0}\right)-2 \alpha V_{2} \text {; } \\
& \dot{V}_{3}=y^{T}(t) G_{1} y(t)-e^{-2 \alpha h_{1}} y^{T}\left(t-h_{1}\right) G_{1} y\left(t-h_{1}\right)-2 \alpha V_{3} \text {. }
\end{aligned}
$$

Then, we have

$$
\begin{aligned}
& \dot{V}(.)+2 \alpha V(.) \\
& \leq y^{T}(t)\left[-P A^{T}-A P\right] y(t)-y^{T}(t) B B^{T} y(t)+2 y^{T}(t) W_{0} f(.) \\
& +2 y^{T}(t) W_{1} g(.)+y^{T}(t)\left(\sum_{i=0}^{1} G_{i}\right) y(t)+2 \alpha\langle P y(t), y(t)\rangle \\
& +\dot{y}^{T}(t)\left(\sum_{i=0}^{1} h_{i}^{2} H_{i}\right) \dot{y}(t)-\sum_{i=0}^{1} e^{-2 \alpha h_{i}} y^{T}\left(t-h_{i}\right) G_{i} y\left(t-h_{i}\right) .
\end{aligned}
$$


Adding $f^{0}(t, x(t), x(t-h(t)), u(t))-f^{0}(t, x(t), x(t-h(t)), u(t))=0$, respectively into (11) and using the condition (4) for the following estimations

$$
\begin{aligned}
f^{0}(t, x(t), x(t-h(t)), u(t)) \leq & \left\langle Q_{1} x(t), x(t)\right\rangle+\left\langle Q_{2} x(t-h(t)), x(t-h(t))\right\rangle \\
& +\langle R u(t), u(t)\rangle \\
= & \left\langle P Q_{1} P y(t), y(t)\right\rangle+\left\langle P Q_{2} P y(t-h(t)), y(t-h(t))\right\rangle \\
& \quad+0.25\left\langle B R B^{T} y(t), y(t)\right\rangle \\
2\left\langle W_{0} f(x), y\right\rangle \leq & \left\langle W_{0} D_{0} W_{0}^{T} y, y\right\rangle+\left\langle D_{0}^{-1} f(x), f(x)\right\rangle \\
2\left\langle W_{1} g(z), y\right\rangle \leq & \left\langle W_{1} D_{1} W_{1}^{T} y, y\right\rangle+\left\langle D_{1}^{-1} g(z), g(z)\right\rangle \\
2\left\langle D_{0}^{-1} f(x), f(x)\right\rangle \leq & \left\langle F D_{0}^{-1} F x, x\right\rangle \\
2\left\langle D_{1}^{-1} g(z), g(z)\right\rangle \leq & \left\langle E D_{1}^{-1} E z, z\right\rangle
\end{aligned}
$$

we obtain

$$
\begin{aligned}
\dot{V}(.)+2 \alpha V(.) \leq & \zeta^{T}(t) \mathcal{E} \zeta(t)+y^{T}(t) S_{1} y(t)+y^{T}(t-h(t)) S_{2} y(t-h(t)) \\
& -f^{0}(t, x(t), x(t-h(t)), u(t))
\end{aligned}
$$

where $\zeta(t)=\left[y(t), \dot{y}(t), y\left(t-h_{0}\right), y\left(t-h_{1}\right), y(t-h(t)), f(),. g().\right]$, and

$$
\begin{gathered}
\mathcal{E}=\left[\begin{array}{ccccc}
W_{11} & W_{12} & W_{13} & W_{14} & W_{15} \\
* & W_{22} & W_{23} & W_{24} & W_{25} \\
* & * & W_{33} & W_{34} & W_{35} \\
* & * & * & W_{44} & W_{45} \\
* & * & * & * & W_{55}
\end{array}\right] \\
S_{1}=-P A-A^{T} P+4 P F D_{0}^{-1} F P+P Q_{1} P, \\
S_{2}=W_{1} D_{1} W_{1}^{T}+4 P E D_{1}^{-1} E P+P Q_{2} P .
\end{gathered}
$$

Note that by the Schur complement lemma, Proposition 1 , the conditions $S_{1}<0$ and $S_{2}<0$ are equivalent to the conditions (7) and (8), respectively. Therefore, by condition $(6),(7),(8)$, we obtain from (12) that

$$
\dot{V}\left(t, x_{t}\right) \leq-2 \alpha V\left(t, x_{t}\right), \quad \forall t \geq 0 .
$$

Integrating both sides of (14) from 0 to $t$, we obtain

$$
V\left(t, x_{t}\right) \leq V(\phi) e^{-2 \alpha t}, \quad \forall t \geq 0 .
$$

Furthermore, taking condition (10) into account, we have

$$
\lambda_{1}\|x(t, \phi)\|^{2} \leq V\left(x_{t}\right) \leq V(\phi) e^{-2 \alpha t} \leq \lambda_{2} e^{-2 \alpha t}\|\phi\|^{2},
$$


then

$$
\|x(t, \phi)\| \leq \sqrt{\frac{\lambda_{2}}{\lambda_{1}}} e^{-\alpha t}\|\phi\|, \quad t \geq 0
$$

which concludes the exponential stability of the closed-loop system (5). To prove the optimal level of the cost function (3), we derive from (12) and (6) (8) that

$$
\dot{V}\left(t, z_{t}\right) \leq-f^{0}(t, x(t), x(t-h(t)), u(t)), \quad t \geq 0 .
$$

Integrating both sides of (14) from 0 to $t$ leads to

$$
\int_{0}^{t} f^{0}(t, x(t), x(t-h(t)), u(t)) d t \leq V\left(0, z_{0}\right)-V\left(t, z_{t}\right) \leq V\left(0, z_{0}\right),
$$

dute to $V\left(t, z_{t}\right) \geq 0$. Hence, letting $t \rightarrow+\infty$, we have

$$
J=\int_{0}^{\infty} f^{0}(t, x(t), x(t-h(t)), u(t)) d t \leq V\left(0, z_{0}\right) \leq \lambda_{2}\|\phi\|^{2}=J^{*} .
$$

This completes the proof of the theorem.

Remark 1. Theorem 1 provides sufficient conditions for guaranteed cost control for a class of Hopfield delayed neural networks (1) in terms of the solutions of LMIs, which guarantees the closed-loop system to be exponentially stable with a prescribed decay rate $\alpha$. The developed method using new inequalities for lower bounding cross terms eliminate the need for over bounding and provide larger values of the admissible delay bound. Note that the time-varying delays are non-differentiable, therefore, the methods proposed in [1-9] are not applicable to system (1). The LMI condition (2) depends on parameters of the system under consideration as well as the delay bounds. The feasibility of the LMIs can be tested by the reliable and efficient Matlab LMI Control Toolbox $[10]$.

\section{Conclusion}

In this paper, the problem of guaranteed cost control for Hopfield neural networks with interval nondifferentiable time-varying delay has been studied. A nonlinear quadratic cost function is considered as a performance measure for the closed-loop system. The stabilizing controllers to be designed must satisfy some exponential stability constraints on the closed-loop poles. By constructing a set of time-varying Lyapunov-Krasovskii functional combined with NewtonLeibniz formula, a memoryless state feedback guaranteed cost controller design 
has been presented and sufficient conditions for the existence of the guaranteed cost state-feedback for the system have been derived in terms of LMIs.

\section{Acknowledgments}

This work was supported by the Thailand Research Fund Grant, the Commission for Higher Education and Faculty of Science, Maejo University, Thailand. The authors thank anonymous reviewers for valuable comments and suggestions, which allowed us to improve the paper.

\section{References}

[1] J.J. Hopfield, Neural networks and physical systems with emergent collective computational abilities, Proc. Natl. Acad. Sci. USA, 79(1982), 2554-2558.

[2] VN. Phat, Y. Kongtham, and K. Ratchagit, LMI approach to exponential stability of linear systems with interval time-varying delays, Linear Algebra Appl., Vol. 436, pp. 243-251, 2012. doi: 10.1016/j.laa.2011.07.016

[3] Kreangkri Ratchagit, STABILITY ANALYSIS OF LINEAR SYSTEMS WITH TIME DELAYS, International Journal of Pure and Applied Mathematics, Vol. 76 No. 1, 2012, 21-28.

[4] K. Ratchagit, Asymptotic stability of delay-difference system of Hopfield neural networks via matrix inequalities and application, International Journal of Neural Systems, 17(2007), 425-430. DOI: 10.1142/S0129065707001263

[5] K. Ratchagit, V.N. Phat, Stability and stabilization of switched linear discrete-time systems with interval time-varying delay, Nonlinear Anal. Hybrid Syst. 5 (2011) 605-612. DOI: 10.1016/j.nahs.2011.05.006

[6] K. Ratchagit , THE SUFFICIENT CONDITIONS FOR STABILITY OF LINEAR TIME-VARYING SYSTEMS WITH STATE DELAYS, International Journal of Pure and Applied Mathematics, Vol. 65 No. 1, 2010, $65-72$.

[7] K. Ratchagit and V.N. Phat, Stability criterion for discrete-time systems, J. Ineq. Appl., 2010(2010), 1-6. doi:10.1155/2010/201459 
[8] Grienggrai Rajchakit, Delay-Dependent Asymptotical Stabilization Criterion of Recurrent Neural Networks, Applied Mechanics and Materials. 330(2013) 1045-1048. doi:10.4028/www.scientific.net/AMM.330.1045

[9] K. Ratchagit, Asymptotic stability of nonlinear delay-difference system via matrix inequalities and application, International Journal of Computational Methods, pp. 389-397, 2009. DOI: 10.1142/S0219876209001899

[10] R.P. Agarwal, Difference Equations and Inequalities, Second Edition, Marcel Dekker, New York, 2000. 
